\title{
Massive gastric dilatation in a patient with type 2 diabetes
}

\section{Andreas Gunter Bach MD, Alexey Surov MD}

Competing interests: None declared.

This article has been peer reviewed.

Affiliation: Department of Radiology, University of Halle, Halle, Germany

\section{Correspondence to:} Andreas Gunter Bach, mail@andreas-bach.de

CMAJ 2013. DOI:10.1503 /cmaj.121524
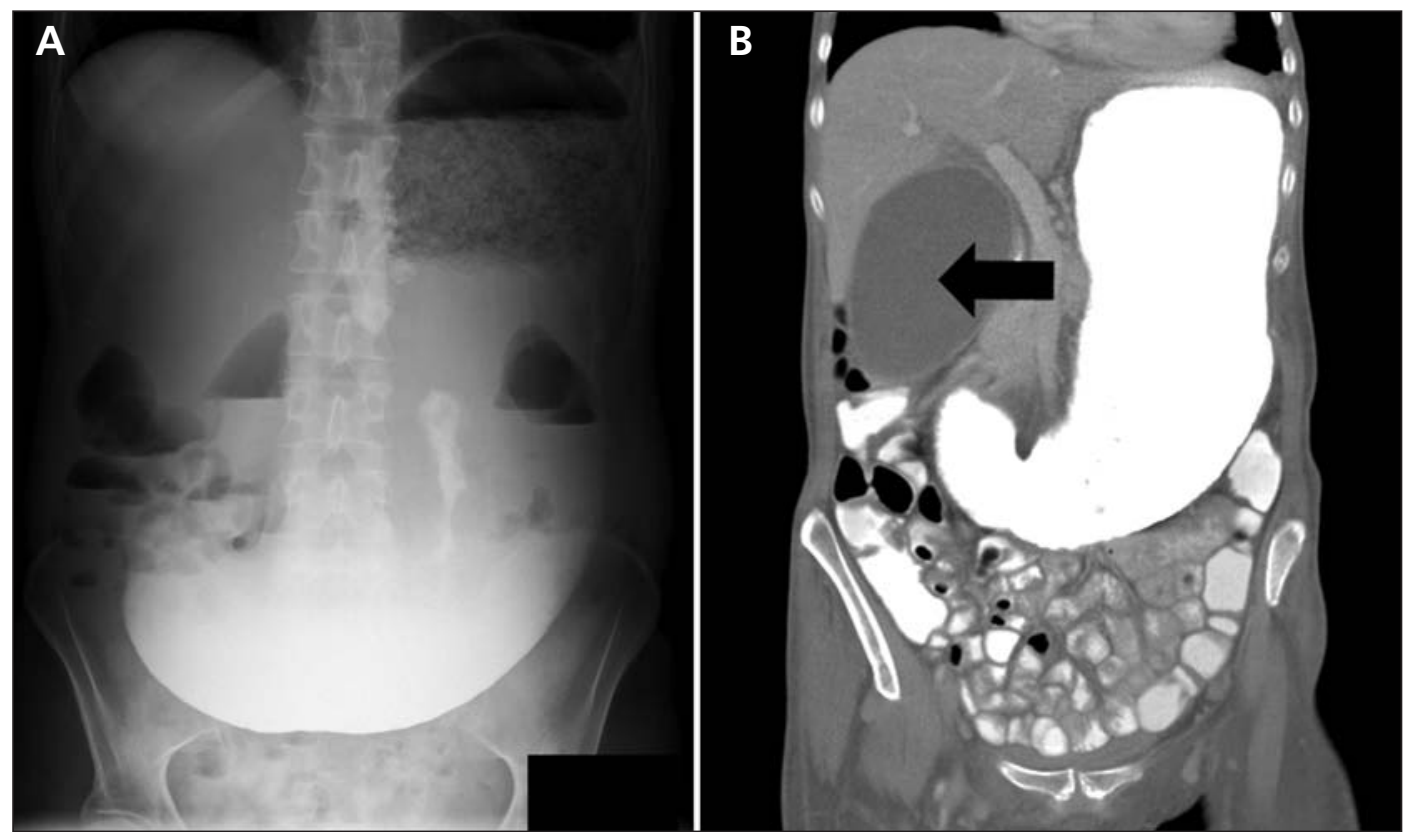

Figure 1: Abdominal radiograph (A) following ingestion of water-soluble contrast media (100 $\mathrm{mL})$, showing pooling in the pelvic region of a 60 -year-old woman with poorly controlled diabetes and severe diabetic gastroparesis. The computed tomograph (B) was obtained shortly after ingestion of the contrast media and shows dilatation of the stomach and gallbladder (arrow).

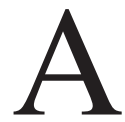
60-year-old woman with long-standing poorly controlled type 2 diabetes mellitus was admitted to hospital following 3 weeks of nausea and nocturnal vomiting, and weight loss of $4 \mathrm{~kg}$. On physical examination, she had a soft abdomen and a palpable gallbladder. Abdominal radiography using water-soluble oral contrast medium (Figure 1) showed pooling in the pelvic region. Computed tomography performed immediately after radiography showed massive dilatation of the stomach and gallbladder (Figure 1). There was no radiographic or endoscopic evidence of neoplasm or stenosis. We diagnosed diabetic gastroparesis.

Major gastric dilatation can be seen in many clinical situations, including a range of dysmotility syndromes, neurologic disorders and connective tissue diseases and in association with neoplasm..$^{1,2}$ The most common cause, however, is diabetic gastroparesis, which occurs in up to $50 \%$ of patients with long-standing type 1 or 2 diabetes. ${ }^{1,2}$ Diabetic gastroparesis is typically much less extreme than in our patient's case. Gastric motility studies are the diagnostic gold standard. ${ }^{2}$

Diabetic gastroparesis can be asymptomatic or it may manifest with postprandial fullness, nonspecific epigastric discomfort and weight loss. The pathophysiology is believed to involve some combination of autonomic neuropathy and dysfunction of Cajal pacemaker cells, although hyperglycemia can also delay gastric emptying. ${ }^{1,2}$

Conservative treatment consists of dietary changes, blood glucose management and the judicious use of promotility agents. ${ }^{2}$ Our patient responded well to this approach, but gastrojejunostomy tubes and gastric pacing devices may be helpful in recalcitrant cases. ${ }^{1,2}$

\section{References}

1. Ma J, Rayner CK, Jones KL, et al. Diabetic gastroparesis: diagnosis and management. Drugs 2009;69:971-86.

2. Hasler WL. Gastroparesis. Curr Opin Gastroenterol 2012;28:621-8. 